\title{
Training of Ecological Detection Dogs for Wolf Scat (Canis lupus)
}

\author{
Hilde VERVAECKE ${ }^{*}$, Ellen VAN KRUNKELSVEN ${ }^{1}$, Koen VAN DEN BERGE²
}

\author{
${ }^{1}$ Agro-and Biotechnology Research Group, Odisee University of Applied Sciences, Hospitaalstraat 21, 9100 Sint-Niklaas, \\ Belgium \\ ${ }^{2}$ Research Institute for Nature and Forest, Havenlaan 88 bus 73, B-1000 Brussels, Belgium \\ * Corresponding author: H. Vervaecke e-mail: hilde.vervaecke@odisee.be
}

\section{RESEARCH ARTICLE}

\begin{abstract}
Detection dogs for wolf scat can assist in conservation-monitoring of wolves. In this pilot project, the first encounters of experienced detection dogs with wolf scat were documented. It was tested how a dog, trained with only a few scat samples (minimally four, maximally six), could generalise towards new unknown wolf scats and discriminate them from distracting odours. Four dogs all showed a behavioural expression of aversion upon first presentation with wolf scat during their initial training to fixate on wolf scat. The dog that showed the least aversion to wolf scats was trained during two weeks. His ability to discriminate wolf scat from other odours and to generalise to new wolf samples was evaluated. He was presented a line-up with new wolf scat, dog scat and other distracting odours in eight boxes, seven trials and random rotations of odours. The test was conducted blindly without the dog handler knowing if or where a positive sample was present. The dog showed partial generalisation of wolf scat odour from only a few known training samples to new wolf samples. The test was evaluated for methodological improvements.
\end{abstract}

Keywords: Canis lupus; scat; detection dogs.

Received: 17 September 2020

Accepted: 14 April 2021

Published: 15 May 2021

DOI:

10.15835/buasvmcn-asb:2020.0021

2021 Authors. The papers published in this journal are licensed under the Creative Commons Attribution-NonCommercialNoDerivatives 4.0 International License

\section{INTRODUCTION}

In several countries, people are training ecological scent dogs to detect a variety of species for surveying and monitoring of wildlife (e.g. Wasser et al., 2004). This proves to be a powerful tool in international mammalian conservation biology (Orkin et al., 2016). The wolf (Canis lupus), a species with a high detection difficulty and high monitoring concern, recently returned to Belgium (Van Den Berge, 2018). Wolf scats are being collected to study identities and diets. Scat detection with scent dogs has proven to be a more efficient method than visual human scanning (Da Silva et al., 2020), with a $30 \%$ higher detection success in the field (Böcker, 2016) and 99,6\% time savings (Roda et al., 2021). Detection of wolf scats by dogs was successfully done in for instance Germany (Pellegrini \& Krummheuer 2016), Portugal (Palacios et al. 2017) or France (Roda et al., 2021). When experienced detection dogs are trained on a new odour, they need to be able to generalise a limited number of training samples to other variants of the target odour caused by sex, age, diet etc. (Oldenburg et al., 2016). The dog must not only recognize but also discriminate the target odour among other more or less similar distractor odours (Gadbois \& Reeve, 2014) such as among scats of related species with similar diets (Grimm-Seyfarth et al. 2019). In the field, detection dogs are thus required to discriminate a target odour from non target odours, showing sufficient specificity, while enabling some degree of variation in the target odours thus showing sufficient generality (Oldenburg et al., 2016). Training with one particular sample of the odour target tends to reduce the tendency to 
generalise to other variants of the target, whereas training with a range of variants can enhance generalisation. Best practices for maximizing generalisation are to train with many exemplars of a target that vary by irrelevant dimensions to generalise to other variants of the trained target (Lazarowski et al., 2020). In some cases, target odours are not easy to obtain, such as scat samples of wolves and other elusive species. Therefore, the question whether some form of generalisation can occur in a dog trained with a limited number of samples can be interesting. Oldenburg et al. (2016) trained a Malinois on only two variations of captive otter spraints and found the dog could generalise to new wild spraints. In a pilot study on otter spraints, two dogs were trained on two otter spraints, one from a captive otter fed on chicken carcasses and one very dry otter spraint from the wild. When the dogs were presented with a new unknown fresh otter spraint from the wild among six unknown distraction odours including scats of pine marten and stone marten, they correctly recognized the target odour (Van Cauteren, 2015). Rutter et al. (2020) found that dogs can effectively generalise their alert behaviour from one target species of stoneflies to another closely related species, without previous exposure. To determine whether generalisation occurred, Lazarowski et al. (2015) proposed to compare responses to the untrained targets and the trained targets. If the number of hits to the test odours is not significantly different than the number of hits to the trained target, it can be concluded that the dogs successfully generalised (Lazarowski et al., 2020). Periodic testing to evaluate progress is a common training practice. In this pilot project, it was examined how a trained ecological search dog - previously trained on other odours - performed after a short training phase with a few wolf scat samples, in a discrimination test containing unknown new wolf scats and other distracting odours, to observe whether some form of generalisation had already occurred. The reaction of four dogs upon the first exposure to wolf scat and the initial training phase is also described. The test is evaluated for methodological improvements.

\section{MATERIALS AND METHODS}

This pilot project consisted of an odour imprinting training on wolf scat and a wolf scat discrimination/generalisation test. Several zoos that keep groups of European wolves provided wolf faecal samples. The exact identity of the scat producing individual was not known.

\section{Odour imprinting training}

In 2018 several volunteers were selected by a professional scent dog trainer to participate at an ongoing project with the Research Institute for Nature and Forest (INBO) (Stockmans et al., 2019). In spring 2019 four experienced dogs (Table 1) participated at a training day in order to imprint on wolf scat as a new target odour.

Table 1. Dogs that participated at the odour imprinting training

\begin{tabular}{|c|c|c|c|c|c|}
\hline Name & Breed & Sexe & Age & Habitual target species & Level \\
\hline Wietse & Flatcoated Retriever & Male & 6 & Stag beetle, hermit beetle & Operational \\
\hline Smoke & Malinois 1 & Female & 4.5 & Stag beetle, otter & Operational \\
\hline Kikki & English Springer Spaniel & Female & 2.5 & European hamster & In training \\
\hline Pippa & Malinois 2 & Female & 4 & Bull frog & In training \\
\hline
\end{tabular}

For this odour imprinting training, three wolf faecal samples were used. One sample came from one zoo, two from another zoo; one was fresh, two had been kept frozen and were thawed. The behavioural reactions of the dogs upon first contact were observed. All dogs had learned to alert passively by fixating i.e. pointing the nose and holding for several seconds at the target sample. To train the dogs to fixate on wolf faeces, one wolf scat sample (about $3 \mathrm{~cm}$ long) was provided in a glass and metal container. The dogs were rewarded when showing an intention of their previously learned pointing behaviour at the container with the wolf scat (Figure 1). This was gradually lengthened to a longer fixation of eight seconds. The dogs were rewarded with food or a toy. Subsequently, when all dogs fixated on the container with wolf scat, a line up with three containers was set up. In one container a wolf sample was put, the other ones were empty. The containers were randomly rotated. In this setting each dog had three or four training sessions of about five minutes each. Each dogs' behavioural reactions were documented upon first exposure to the wolf scat. All dogs fixated slower than usual on the wolf sample and all continued to show some aversion to the wolf 
scat sample. At this point it was decided to proceed to a next setting only with the dog showing least aversion to the wolf scat.

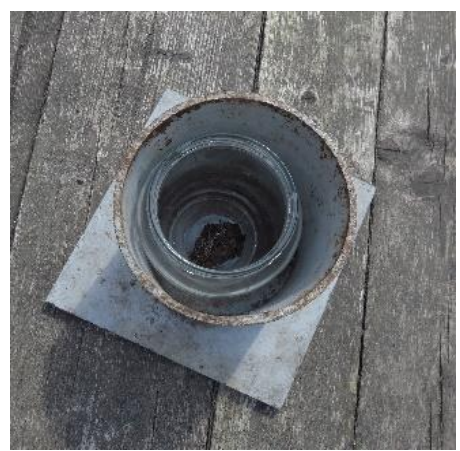

Figure 1. Glass and metal container with wolf scat sample

This was a line up with eight metal stainless steel boxes (as seen in Figure 2 but located outdoors), only one of them containing a wolf sample. The dog was trained by rewarding him with a thrown toy as soon as he passed and sniffed at the wolf sample. This was repeated four times before he spontaneously fixated during six consecutive trials on the wolf sample at any location in the line-up. No distracting odours were offered at this stage.

After that training day, the volunteers were asked to continue to train the dogs at home on the scent discrimination phase during the next two weeks. Each volunteer received three wolf scat samples to take home, two of which were the same as on the odour imprinting training or came from the same zoo, and one coming from a new zoo. Three of the four dogs continued to show markedly low enthusiasm when training on the wolf samples and the owners decided not to continue the training. They did not want to risk that their dogs' motivation and pleasure during detection training would be negatively affected. Only the Flatcoated Retriever continued to show high enthusiasm. He was trained daily at first with any of the three samples and a variety of distracting odours, food such as tea or coffee, gloves, etc. in a line up of eight boxes 6 or 10 times twice a day. He was rewarded either by a toy thrown at him by a helper who assisted the handler or by clicking followed by a "treat and train" food dispenser. Several blank trials without wolf samples were included. At first the dog was rewarded immediately after very brief fixating behaviour. Then gradually, the length of fixating was increased up to eight seconds. This was continued until each of the three samples could be found $100 \%$ correctly among various distracting odours in eight metal containers, in at least six consequent randomized line-ups. After this home pre-test, he was ready for the discrimination/generalisation test with unfamiliar odours. On the initial training day, he had been exposed to three scats coming from two zoos. During the home training period, he was exposed to three scats, one of which came from another zoo, two of which could have been the same scats as on the initial training day. In total he was thus exposed to minimally four to maximally six different scats, coming from minimally two to maximally six different individuals.

\section{Discrimination/generalisation test}

It was tested if the dog could recognize new wolf scat samples from other distracting odours. The dog had to discriminate the wolf odour from other odours and to generalise the odour from the previously used training samples to the newly presented unknow wolf samples. This type of test is a common practice during detection training to evaluate the dogs' performances. Three new wolf scat samples were used, coming from three different zoos. The target (wolf scat samples) and non-target odours (the distracting odours) had not been presented previously to the dog. The dog was presented with a line-up with eight metal boxes containing a plastic holder with the samples, with seven trials and random rotation of the boxes.

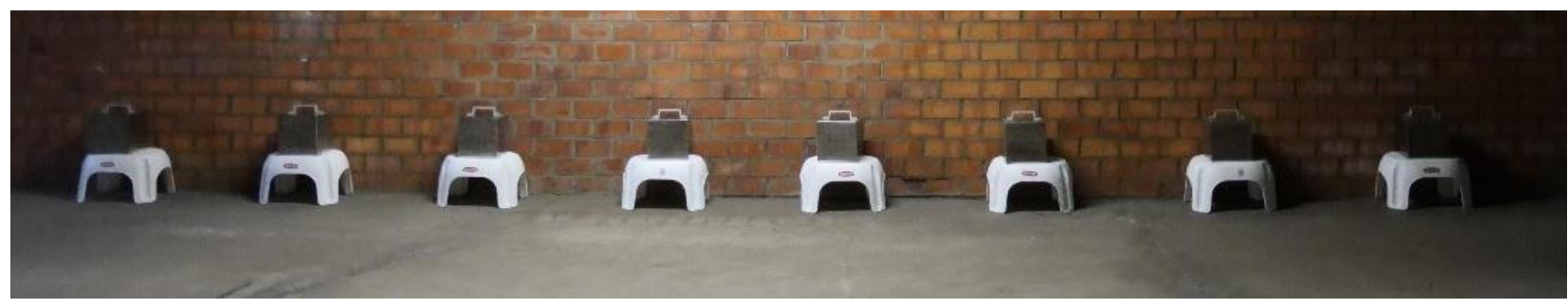

Figure 2. Line-up of eight metal stainless steel boxes

It was impossible to see the content of the boxes from the outside. The distracting odours were five different dog scats, sheep scats, chicken scat, horse scats, stones, lion's mane mushroom, one scat from a dog fed on entire rabbit carcasses. In five trials there was one target sample (wolf scat) and seven non-target distracting odours or an occasional empty pot. In trial 1, wolf sample 1 was used; in trial 2 and 3, wolf sample 2 was used; in trial 4 and 5 , wolf sample 3 was used. Two trials (trial 6 and 7) were a "negative" line-up with eight non-target distracting odours, 
without any wolf scat. The dog was led to the left of the line-up by the dog's owner, unleashed and given the command to search. The dog passed the line from left to right and he was allowed to walk along independently twice or three times at a maximum (Grimm-Seyfarth et al., 2019). The test was conducted blindly without the dog owner knowing if and where a positive sample was placed in the line-up and whether the line-up contained a positive sample. The handler told the experimenter when the dog was alerting to a target. The dog was rewarded by the handler after a verbal confirmation had been given by the experimenter whether the dog was correct. When the dog had found a target early in the line, he was rewarded verbally, asked to continue to snif the rest of the line and rewarded with his toy.

It was scored whether the handler said the dog was fixating at the target (wolf scat) or non-target samples (distracting odours or empty pot), and when the handler said the dog showed interest without fixating. The fixating scores only were classified as true positives (alert, target odour present), true negatives (no alert, no target odour present), false positives (alert, no target odour present) or false negatives (no alert, target odour present). Mosconi et al. (2017) describe the performance of the dog in correctly locating the target as the overall percentage of correct indications and the percentage of correctly detected targets for the total number of targets. They suggest using operational measures defined by Allouche et al. (2006) (see Table 2).

Table 2. Definition of detection performance indicators i.e. accuracy, sensitivity and specificity and operational measures, adapted from Allouche et al. (2006)

\begin{tabular}{|c|c|c|}
\hline Accuracy & overall proportion of correct indications & CPS+CNS/NT $\dagger$ \\
\hline Sensitivity & proportion of correct positive indications & CPS/TT (i.e. CPS+TND) $\dagger$ \\
\hline Specificity & proportion of correct negative indications & CNS/NR (i.e. CNS+TWD) $\dagger$ \\
\hline
\end{tabular}

†CPS: correct positive signaling: total $n^{\circ}$ of pots with wolf scat present in line-ups and correctly detected and signaled by the dog; CNS: correct negative signaling: total $n^{\circ}$ of pots without wolf scat and not signaled; NT: total $n^{\circ}$ of pots investigated; TT: total $n^{\circ}$ of wolf scats present in the pots; TND: target not detected: total $n^{\circ}$ of wolf scats present in the pots and not detected; NR: no reaction; TWD: targets wrongly detected: total $n^{\circ}$ of pots without target signaled by the dog as if the target was present.

\section{RESULTS}

\section{Odour imprinting training}

During the initial imprinting on the target odour the dogs' reactions were observed. Behaviourally, all dogs showed an aversion when a wolf scat sample in a glass container was first presented to them. Malinois dog 1 backed away and was reluctant to come near the sample or to put her nose close to the sample. Malinois dog 2 was reluctant to approach, averting her head. After some rewards for sniffing she would fixate but always lift her back foot at the same time, a behaviour not shown during her usual scent training on bull frogs. The Springer Spaniel avoided going to the pot and avoided putting her nose above the sample. The Flatcoated Retriever showed somewhat less enthusiasm than usual and was slower than usual in learning to fixate on the new odour. For all the dogs, their initial behaviour differed from their usual behaviour when being trained for other odours.

Subsequently, the target odour was presented in one pot (wolf scat sample) next to two empty pots:

- Malinois 1: The aversion remained. In a line-up with two or three pots, she prefered to point at all the other samples instead of the wolf scat. She systematically did not approach the wolf sample, which can be read as an alternative indication in itself. Fixating on the wolf sample improved with an extra toy as a reward. After several trials and rewards of brief correct pointing, she started to point directly at the wolf sample still keeping more distance than during a normal positive fixation.

- Malinois 2: She continued to avoid the wolf sample. The dog was rewarded for briefly pointing over several trials until she started to point correctly at the wolf sample. She repeatedly lifted her back foot while fixating with a long neck.

- English Springer Spaniel: She showed little interest in the wolf sample and she continued to search at other locations. She did not participate at the trials with three pots, first needing to fixate better prior to taking the next step.

- Flatcoated Retriever: He was first trained by associating the wolf odour with his toy, that was thrown at him as he passed by the correct pot. After a couple of trials, he correctly stopped at the wolf odour and pointed. He also showed increasing enthusiasm and tail wagging as the number of correct fixations increased. He showed the strongest progression although he seemed to progress somewhat slower than usual.

Overall, after several rewards for approximations of the behaviour, three of the four dogs showed correct pointing behaviour at the wolf scat in the set-up with three pots. 


\section{Discrimination/generalisation test}

Only the Flatcoated Retriever performed this test as the other dog owners had not continued the training due to the low enthusiasm of their dogs when training with wolf samples. During the test, seven trials were performed and the results were scored. In the first trial the dog was presented with a line up in which the wolf target sample had never been encountered before. All other samples were new as well, except for one known dog sample. There was an empty pot, a new wolf scat sample of an unknown wolf (wolf scat 1), a new dog faecal sample (dog scat 1), another new dog faecal sample (dog scat 2), a sheep faecal sample, a stone, a lion's mane mushroom and a known dog faecal sample (dog scat 3). He correctly fixated on the wolf scat.

In the second trial, he was presented with a new dog faecal sample (dog scat 4), and a new dry wolf faecal sample (wolf scat 2). Coming to this dry sample, he sniffed longer, then went further and came back to point and fixate at the wolf scat sample.

In the third trial, the samples of the previous trial were randomized. The dog showed interest at the wolf scat sample (wolf scat 2) but did not give a fixation at any sample.

In the fourth trial, he was presented with a fresh wolf scat sample (wolf scat 3) and distracting odours with a new dog scat. He fixated on the wolf scat.

In the fifth trial, a similar line up was repeated with the same wolf scat sample (wolf scat 3) and a faecal sample of a dog that ate a carcass of a rabbit. Instead of another dog scat, a chicken scat was added to the distracting odours. He fixated correctly on the wolf scat sample after passing and checking the dog scat sample.

In the sixth trial, a partly similar line up was offered, this time without any wolf sample (a "negative run"). He passed by the carcass-fed dog scat sample and searched further, without fixating anywhere.

Then, in the seventh trial, the exact same trial was repeated. This time, he did fixate on the carcass-fed dog scat sample, incorrectly.

Over all seven trials, the dog performed with an accuracy of 97\% (CPS+CNS/NT), sensitivity of 80\% CPS/TT (i.e. CPS+TND) and specificity of $98 \%$ (CNS/NR (i.e. CNS+TWD)). He made one false alert (TWD) and missed one target (TND) (cfr. Table 3).

Table 3. Performance of the dog during the discrimination/generalisation test trials

\begin{tabular}{|c|c|c|c|c|c|c|c|}
\hline Trial & $\begin{array}{c}\text { Correct } \\
\text { positive } \\
\text { (CPS } \dagger \text { ) }\end{array}$ & $\begin{array}{c}\text { Correct } \\
\text { negative } \\
\text { (CNS } \dagger \text { ) }\end{array}$ & $\begin{array}{c}\text { Total pots } \\
\text { (NT } \dagger \text { ) }\end{array}$ & $\begin{array}{c}\text { Total } \\
\text { targets } \\
\text { (TT } \dagger \text { ) }\end{array}$ & $\begin{array}{c}\text { Targets not } \\
\text { found } \\
\text { (TND } \dagger \text { ) }\end{array}$ & $\begin{array}{c}\text { No reaction } \\
\text { (NR } \dagger \text { ) }\end{array}$ & $\begin{array}{c}\text { Targets } \\
\text { wrong } \\
\text { (TWD } \dagger \text { ) }\end{array}$ \\
\hline $\mathbf{1}$ & 1 & 7 & 8 & 1 & 0 & 7 & 0 \\
\hline $\mathbf{2}$ & 1 & 7 & 8 & 1 & 0 & 7 & 0 \\
\hline $\mathbf{3}$ & 0 & 7 & 8 & 1 & 1 & 8 & 0 \\
\hline $\mathbf{4}$ & 1 & 7 & 8 & 1 & 0 & 7 & 0 \\
\hline $\mathbf{5}$ & 1 & 7 & 8 & 1 & 0 & 7 & 0 \\
\hline $\mathbf{6}$ & 0 & 8 & 8 & 0 & 0 & 8 & 0 \\
\hline $\mathbf{7}$ & 0 & 7 & 8 & 0 & 0 & 7 & 1 \\
\hline SUM & 4 & 50 & 56 & 5 & 1 & 51 & 1 \\
\hline
\end{tabular}

†CPS: correct positive signaling: total $\mathrm{n}^{\circ}$ of pots with wolf scat present in line-ups and correctly detected and signaled by the dog; CNS: correct negative signaling: total $n^{\circ}$ of pots without wolf scat and not signaled; NT: total $n^{\circ}$ of pots investigated; TT: total $n^{\circ}$ of wolf scats present in the pots; TND: target not detected: total $n^{\circ}$ of wolf scats present in the pots and not detected; NR: no reaction; TWD: targets wrongly detected: total $\mathrm{n}^{\circ}$ of pots without target signaled by the dog as if the target was present.

The dog did not fixate randomly, showing 4 alerts on 5 target odours and 1 alert on 51 non-target odours, demonstrating a correct alert rate above chance level $(\mathrm{P}<0.0001$; two tailed Fisher exact test).

\section{DISCUSSION}

When wolf scat was first presented, all the dogs in this pilot-project showed an aversive reaction, possibly a sign of conflict behaviour due to the aversive odour. This aversion was speculated to be an adaptation to avoid exposure to faecal-borne intestinal parasites and pathogens (Hart et al., 2012; Hart et al., 2018), or could be an aversion to a potential predator (Mech, 1970) or competitor (Lescureux and Linnell, 2014). When selecting a wolf scat detection dog, a high drive and high motivation to overcome a possible aversion, may be part of the criteria.

After a further pilot training of only one dog, he showed the ability to partially generalize to new wolf scats after exposure to a few wolf scat samples (minimally four, maximally six) over a period of two weeks. In a test with seven 
trials, he correctly discriminated three new wolf scat samples (true positives) from distracting odours, including carcass-fed dog faeces. He showed one false negative, missing a very dry wolf sample. He showed one false positive during a blank trial by fixating at a carcass-fed dog scat sample. Oldenburg et al. (2016) proposed a generalisation training for wildlife detection dogs with a stepwise testing procedure and pre-set performance criteria allowing to determine how much odour variation is needed for generalisation and concept formation to occur. Grimm-Seyfarth et al. (2019) suggest that dogs have to pass a pre-test with $100 \%$ accuracy to be sure sufficient generalisation occurs prior to continue with discrimination training. In the pilot project, an easily set up discrimination test was performed, as periodically used during training in order to test the progress. New wolf samples were presented and the performance of the dog was compared during the test to the performance during training at home with the three known wolf scat samples. The accuracy shown by the dog with the new untrained samples (96\%) was lower than the accuracy of $100 \%$ during the pre-test at home with the training targets. Yet, there was a significantly better performance than chance level. Such a response below baseline training hit rate but above chance, can indicate that some degree of partial generalisation occurred (Lazarowski et al. 2015).

Obviously, a sample size of one in the discrimination and generalisation tests is quite small and only limited conclusions can be drawn from this pilot study. The project does allow for an interesting evaluation of a common testing practice during training, in the light of current scientific knowledge. Several methodological concerns can be addressed.

The dog and the handler were blind to the target location while the experimenter observed each trial (i.e. single blind). However, a double-blind design where neither the dog and handler nor an observer are aware of the target location would be preferred (Johnen et al., 2017).

As the same wolf scat samples were presented in two subsequent trials, a possible learning effect from a previous trial may have occurred, since dogs can show rapid within-session learning after repeated exposures if responses on test trials are reinforced (Gazit et al., 2005). Consecutive repetition of a relatively similar line-up with the same wolf scat or a repetition of the line-up without any wolf scat, seemed to instill doubt in the dog, possibly a bias caused by the design, pointing at an aspect to remediate in the design and in further training (Lazarowski et al., 2020; Johnen et al., 2017). Jezierski et al. (2008) suggest to train on eventual positional biases in dogs by quasirandomisation during training before testing with a fully randomised test.

To test generalisation capacities, the dog's performance should be considered only when encountering new wolf scat samples with different qualities for the first time. This limits the number of trials to three instead of seven. In these first three encounters, the dog correctly identified all the wolf scat samples, but the low number of trials lowers the power to make firm conclusions. An empty distractor container was used in several line-ups. Counting them as distractor odours can artificially increase the success rates. Lastly, the exact same line-up without wolf odour was repeated twice.

It is suggested to include distractor odours from similar and differing chemical and odour categories (Lazaroswki et al., 2020). Apart from the dog scat samples, the distractor odours that were used were relatively dissimilar such as sheep or chicken scat, mushrooms and stone. Increasing the decoy quality by including distractor odours from other carnivores such as foxes, martens, cats, etc. will improve the external validity of the test as predictor of field performance. Also, as some of the samples were randomly reused in the trials, the number of newly encountered distractor odours was limited. Porritt et al. (2015) describe a user-friendly validated procedure for a discrimination test, consisting of about 22.5 interferent odours per target type, whereas only 12 new distracting samples were used in this pilot project, thus lowering the reliability of the test. When testing for discrimination capacities, they suggest to use a pass criterion of at least a $70 \%$ higher hit rate than the false alarm rate. Dogs must respond to their trained target significantly more often than they give a false alarm in order to meet the criterion. In our test, with a correct positive signalling rate (CPS) of $4 / 5$ or $80 \%$ and a false hit rate of $1 / 7$ or $14 \%$, the dog did not reach this discrimination criterion. Obviously, the accredition of the dog was not the target of this pilot study, and more training is required prior to testing for accredition. During the test, the selected dog made some informative mistakes. In the last trial of the negative line-up without any wolf sample, he fixated on the scat of a carcass fed dog among the other distracting odours, although he had not fixated in the same line-up just previously and he had neglected this sample when the wolf scat was present. He thus showed a false positive, indicating the target as present whereas it was absent. This underlines the importance of training on negative line-ups. In the trial with a very dry wolf faecal sample and the other distracting odours, he showed a false negative i.e. he indicated the target was absent whereas it was present, although he had indicated it correctly in the previous line up.

The test difficulty should be increased after a longer period of training with more difficult odours in a follow-up project to ascertain that the dog can ignore carcass-fed dog samples in a trial without the presence of a target wolf sample, in trials with multiple different new wolf, dog and wolfdog, and other carnivore scat samples showing a variation in dryness and age of scat, taking into account the natural faecal mean persistence time (Da Silva et al., 2020) and a variation in the diet of the scat-producing individual. It may also be interesting to include sniffing duration to allow to distinguish between true negatives at which dogs sniff shorter than at false negatives, true positives, and false positives on the other hand (Concha et al, 2014). A change of context can have negative effects on detection performance (Gazit et al., 2005). Therefore this evaluation to generalize to new scats in a controlled environment should also be done in a field context. 
Author Contributions: H.V., E.V. and K.V. conceived the study; E.V. was the main trainer-coach in this project and designed the training and test; E.V. and H.V. collected the data; H.V. performed the analysis and wrote the paper with suggestions of E.V. and K.V.

Funding Source: This research was not funded by an external grant. It was funded as pilot project by Odisee University of Applied Sciences ("Voorbereidingsproject Ecologische Zoekhond").

\section{Acknowledgments}

We kindly thank the volunteer Carina De Pape who trained the Flatcoated Retriever Wietse, as well as the other volunteers who participated at the training: Guido Franssen, Jurgen David, Liesbet Brasseur. We thank Arno Thomaes from the Research Institute for Nature and Forest (INBO) who coordinated the scent-dog volunteer project. We thank the collaborating zoos for providing samples. We thank Bente Stockmans for her assistance. We thank the zoos for their provision of samples (La Roche en Ardenne, Forestia, Gaia Zoo, Pairi Daiza, Pakawi Parc, Wolf Center Bilstain).

\section{Conflicts of Interest}

The authors declare that they do not have any conflict of interest.

\section{REFERENCES}

1. Allouche 0 , Tsoar A, Kadmon R. Assessing the accuracy of species distribution models: prevalence, kappa and the true skill statistic (TSS). J Appl Ecol. 2006; 43:1223-1232. https://doi.org/10.1111/j.13652664.2006.01214.x

2. Böcker, F. Sign Survey, Camera Trapping, Scent Detection Dog - Evaluation of different methods to investigate wolf presence. Master thesis, Faculty of Environment and Natural Resources, Albert-Ludwigs University Freiburg im Breisgau, Germany; 2016.

3. Concha A, Mills DS, Feugier A, Zulch H, Guest C, Harris R, Pike TW. Using sniffing behavior to differentiate true negative from false negative responses in trained scent-detection dogs. Chem Senses. 2014; 39(9):749-754. doi:10.1093/chemse/bju045

4. Da Silva AR, De Oliveira ML, Duar JMB. Incorporating the sampling effectiveness of detection dogs in the faecal standing crop method. Eur J Wildl Res. 2020; 66:47. http://hdl.handle.net/11449/198945

5. Gadbois, S, and C Reeve. Canine olfaction: scent, sign, and situation. Pages 3-29 in A. Horowitz, editor. Domestic dog cognition and behavior: the scientific study of Canis familiaris. Springer, Berlin Heidelberg, Germany; 2014.

6. Gazit I, Goldblatt A, Terkel J. The role of context specificity in learning: the effects of training context on explosives detection in dogs. Anim Cogn. 2005; 8:143-50. doi: 10.1007/s10071-004-0236-9.

7. Grimm-Seyfarth A, Zarzycka A, Nitz T, Heynig L, Weissheimer N, Lampa S, et al. Performance of detection dogs and visual searches for scat detection and discrimination amongst related species with identical diets. Nat Conserv. 2019; 37:81-98. https://doi.org/10.3897/natureconservation.37.48208.

8. Hart BL. Behavioral defenses in animals against pathogens and parasites: parallels with the pillars of medicine in humans. Philos Trans R Soc Lond, B, Biol Sci. 2012; 366:3406-3417. https://doi.org/10.1098/rstb.2011.0092

9. Hart BL, Lynette A, Thigpen AP, Tran A, Bain MJ. The paradox of canine conspecific coprophagy. Vet Med Sci. 2018; 4 (2):106-114. doi: 10.1002/vms3.92.

10. Jezierski T, Walczak M, Górecka A. Information-seeking behavior of sniffer dogs during match-to-sample training in the scent lineup. Polish Psychol Bull. 2008; 39:71-80. doi: 10.2478/v10059-008-0010-y.

11. Johnen D, Heuwieser W, Fischer-Tenhagen C. An approach to identify bias in scent detection dog testing. Appl Anim Behav Sci. 2017; 189:1-12. https://psycnet.apa.org/doi/10.1016/j.applanim.2017.01.001.

12. Lazarowski L, Foster ML, Gruen ME, Sherman BL, Fish RE, Milgram NW, et al. Olfactory discrimination and generalisation of ammonium nitrate and structurally related odorants in Labrador retrievers. Anim Cogn. 2015; 18:1255-65. doi: 10.1007/s10071-015-0894-9.

13. Lazarowski L, Krichbaum S, De Greeff LE, Simon A, Singletary M, Angle C, et al. Methodological Considerations in Canine Olfactory Detection Research. Front Vet Sci. 2020; 7:408. doi: 10.3389/fvets.2020.00408.

14. Lescureux N, Linnell JDC. Warring brothers: The complex interactions between wolves (Canis lupus) and dogs (Canis familiaris) in a conservation context. Biol Conserv. 2014; 171:232-245. https://doi.org/10.1016/j.biocon.2014.01.032. 
15. Mech LD. The Wolf. Natural History Press, New York; 1970.

16. Mosconi F, Campanaro A, Carpaneto GM, Chiari S, Hardersen S, Mancini E, et al. Training of a dog for the monitoring of Osmoderma eremita. In: Carpaneto GM, Audisio P, Bologna MA, Roversi PF, Mason F (Eds) Guidelines for the monitoring of the saproxylic beetles protected in Europe. Nat Conserv. 2017; 20:237264. https://doi.org/10.3897/natureconservation.20.12688.

17. Oldenburg C, Schoon A, Heitkönig, IMA. Wildlife detection dog training: A case study on achieving generalisation between target odor variations while retaining specificity. J Vet Behav. 2016; 13:34-38, ISSN 1558-7878, https://doi.org/10.1016/j.jveb.2016.03.008.

18. Orkin J, Yang Y, Yang C, Yu DW, Jiang X. Cost-effective scat-detection dogs: unleashing a powerful new tool for international mammalian conservation biology. Sci Rep. 2016; 6, 34758. https://doi.org/10.1038/srep34758.

19. Palacios V., Garcia E.J., Santos R., Borges C. \& Simões F. Action D.3: Assessment of wolf presence in expansion areas in Portugal: Evaluating the effectiveness of the scat detection dog team. Final Report (Ribeiro S. \& Petrucci-Fonseca F. Coord.); 2017.

20. Pellegrini V. and Krummheuer Y. Etablierung von Spürhunden als effizientes Instrument des Wolfsmonitorings in Deutschland. Project LIFE MedWolf (LIFE11NAT/IT/069). Grupo Lobo/INIAV/FCUL, Lisbon, 22 p. WWF Deutschland; 2016.

21. Porritt F, Mansson R, Berry A, Cook N, Sibbald N, Nicklin S. Validation of a short odour discrimination test for working dogs. Appl Anim Behav Sci. 2015; 165:133-42. doi: 10.1016/j.applanim.2014.11.021.

22. Roda F, Sentilles J, Molins C, Duchamp C, Hansen E, Jean N. Wolf scat detection dog improves wolf genetic monitoring in new French colonized areas. J Vertebr Biol. 2021; 69(3). https://doi.org/10.25225/jvb.20102.

23. Rutter N, Mynott J, Howell T, Stukas A, Pascoe J, Bennett P. et al. Buzzing with possibilities: Training and olfactory generalization in conservation detection dogs for an endangered stonefly species. Aquat Conserv. 2021; 10.1002/aqc.3531.

24. Stockmans B, Thomaes A, Van Krunkelsven E, Vervaecke H. Sniffing for nature. Detection dog training in real life environment. Fourth Natura 2000 Monitoring workshop, 2019 09-11 April, Donana, Spain.

25. Van Cauteren D. De opleiding van een ecologische zoekhond. Bachelorproef Agro- en biotechnologie Dierenzorg, Katholieke Hogeschool Vives, Roeselare; 2016.

26. Van Den Berge K. Year(s) of the wolf: no doubt about it. Lutra (Leiden), 2018; 61(2): 215-217.

27. Wasser SK, Davenport B, Ramage ER, Hunt KE, Parker M, Clarke C et al. Scat detection dogs in wildlife research and management: Application to grizzly and black bears in the Yellowhead Ecosystem, Alberta, Canada. Can J Zool. 2004; 82(3):475-492. 